\title{
Gaussian pulse propagation in coronal loops
}

\author{
César A. Mendoza-Briceño ${ }^{1}$, Leonardo Di G. Sigalotti ${ }^{2}$, \\ and Jordan A. Guerra ${ }^{3}$ \\ ${ }^{1}$ Centro de Física Fundamental, Facultad de Ciencias, \\ Universidad de los Andes, Merida-Venezuela \\ email: cesar@ula.ve \\ ${ }^{2}$ Instituto Venezolano de Investigaciones Científicas (IVIC), Centro de Física, Laboratorio de \\ Física de Fluidos y Plasmas, Caracas-Venezuela \\ email: leonardo.sigalotti@gmail.com \\ ${ }^{3}$ Universidad de Carabobo, Facultad de Ciencias y Tecnología, Departamento de Física, \\ Valencia-Venezuela \\ email: jordan.guerra@gmail.com
}

\begin{abstract}
We study the linear evolution of a Gaussian pulse injected at different locations along a one-dimensional (1D), hot $(T \geqslant 6.3 \mathrm{MK})$ coronal loop, including the dissipative effects of thermal conduction, viscosity, heating, and radiative cooling. We consider both homogeneous and stratified loops of different lengths $(50 \leqslant L \leqslant 400 \mathrm{Mm})$ and values of the pulse width (or standard deviation, $\beta_{g} / L$ ) between 0.005 and 0.02 . We find that a Gaussian velocity pulse can generate propagating waves whose amplitudes increase with increasing width of the pulse. The shape of the waves is quite irregular owing to the superposition of the several harmonics composing the Gaussian pulse. Wave damping due to the combined effects of thermal conduction and viscosity is faster in the shortest and hottest loops. The decay times and periods of the waves are within the observed values of decaying modes of hot SUMER loop oscillations.
\end{abstract}

Keywords. Sun: corona, Sun: oscillations, (magnetohydrodynamics:) MHD, waves

\section{Introduction}

The damping of MHD oscillations and waves (fast or slow) in coronal loops is of great interest because they are natural carriers of energy and possible sources for heating of the coronal plasma and for solar wind acceleration (Banerjee et al. 2007). Recent numerical hydrodynamics simulations of wave dissipation in hot coronal loops have shown results that agree with the observations, predicting periods and damping times close to SoHO and TRACE measurements. In particular, Sigalotti, Mendoza-Briceño \& LunaCardozo (2007) studied the dissipation of an initial velocity perturbation of the form $V_{0} \sin (2 \pi s / L)$, using $1 \mathrm{D}$ hydrodynamical models of hot coronal loops $(T>6.3 \mathrm{MK})$. They found that for typical conditions of the hot corona, thermal conduction increases the periodicity of damped oscillations over the sound crossing time, while the decay times are mostly shaped by the effects of viscosity. Also, they found that under the effects of gravity, nonlinear viscous dissipation leads to a reduction of the decay time compared to the homogeneous case.

Nevertheless coronal loops are often perturbed by nearby flares (Taroyan et al. 2007). In this case, the pertubation is highly localized and the waves (standing and propagating) are generated impulsively. In this work, we study the impulsive generation and dissipation of such waves by initially perturbing the loop with a localized Gaussian velocity distribution and consider the effects of injecting the pulse at different locations along the loop length. 


\section{Governing Equations and Loop Models}

In the solar corona the $\beta$-plasma is much less than 1 and the velocities are less than the Alfvén velocity. As a consequence, the plasma is confined along the magnetic field lines and the loop can be approximately modelled as a 1D flux tube. Thus the assumption is made that the mass flow and heat conduction occurs primarily along the field lines. The evolution can therefore be determined by the usual 1D hydrodynamic equations, with the variable $s$ denoting the position along the loop. Under these assumptions, the equations that govern the dynamics, including the effects of thermal conduction, viscosity, and radiative cooling and heating, are:

$$
\begin{aligned}
\frac{\partial \rho}{\partial t}+\frac{\partial(\rho v)}{\partial s} & =0 \\
\frac{\partial(\rho v)}{\partial t}+\frac{\partial\left(\rho v^{2}\right)}{\partial s} & =-\frac{\partial p}{\partial s}+\rho g(s)+F_{\eta}, \\
\frac{\partial(\rho T)}{\partial t}+\frac{\partial(\rho v T)}{\partial s} & =-\frac{\mu(\gamma-1)}{R_{g}}\left[p \frac{\partial v}{\partial s}+\rho^{2} Q(T)-H-E_{\kappa}-E_{\eta}\right],
\end{aligned}
$$

where $t$ is time, $s$ is the distance along the loop, $\rho$ is the mass density, $v$ is the velocity, $T$ is the plasma temperature, $\mu(=0.6)$ is the mean molecular weight, $\gamma(=5 / 3)$ is the ratio of specific heats, $R_{g}$ is the gas constant, and $p=R_{g} \rho T / \mu$ is the gas pressure. The terms $F_{\eta}, E_{\eta}$, and $E_{\kappa}$ on the right-hand sides of Equations (2.2) and (2.3) represent the viscous forces, the viscous heating, and the conductive heating, respectively, given by

$$
\begin{aligned}
F_{\eta} & =\frac{4}{3} \frac{\partial}{\partial s}\left(\eta \frac{\partial v}{\partial s}\right), \\
E_{\eta} & =\frac{4}{3} \eta\left(\frac{\partial v}{\partial s}\right)^{2}, \\
E_{\kappa} & =\frac{\partial}{\partial s}\left(\kappa \frac{\partial T}{\partial s}\right),
\end{aligned}
$$

where $\eta$ is the coefficient of viscosity defined as (e.g., Braginskii, 1965)

$$
\eta=\frac{0.72\left(m_{p} k_{B}^{5}\right)^{1 / 2}}{\pi^{1 / 2} e^{4} \ln \lambda} T^{5 / 2},
$$

in units of $\mathrm{g} \mathrm{cm}^{-1} \mathrm{~s}^{-1}$, and $\kappa=10^{-6} T^{5 / 2} \mathrm{ergs} \mathrm{cm}^{-1} \mathrm{~s}^{-1} \mathrm{~K}^{-1}$ is the coefficient of thermal conduction along the magnetic field lines (Braginskii, 1965). In Equation (2.7), $m_{p}$ is the proton mass, $k_{B}$ is the Boltzmann constant, $e$ is the electron charge, and $\ln \lambda \approx 23$ is the Coulomb logarithm. The gravitational acceleration term on the right-hand side of Equation (2.2) is assumed to depend only on distance along the loop and is given by

$$
g(s)=-g_{\odot} \cos \left(\frac{\pi s}{L}\right),
$$

where $g_{\odot} \approx 2.74 \times 10^{4} \mathrm{~cm} \mathrm{~s}^{-2}$ is the solar surface gravity and $L$ is the loop length. Implicit in this form is a magnetic field line of perfect semicircular shape and zero inclination angle at the footpoints, with $g(0)=-g_{\odot}$ and $g(L)=g_{\odot}$ on the right and left footpoint, respectively, and $g(L / 2)=0$ at the symmetric loop top.

The radiative cooling is quantified by the optically-thin radiation-loss function $Q(T)$, which is approximated by a piecewise continuous function of the form

$$
Q(T)=\frac{\chi}{m_{p}^{2}} T^{\alpha},
$$



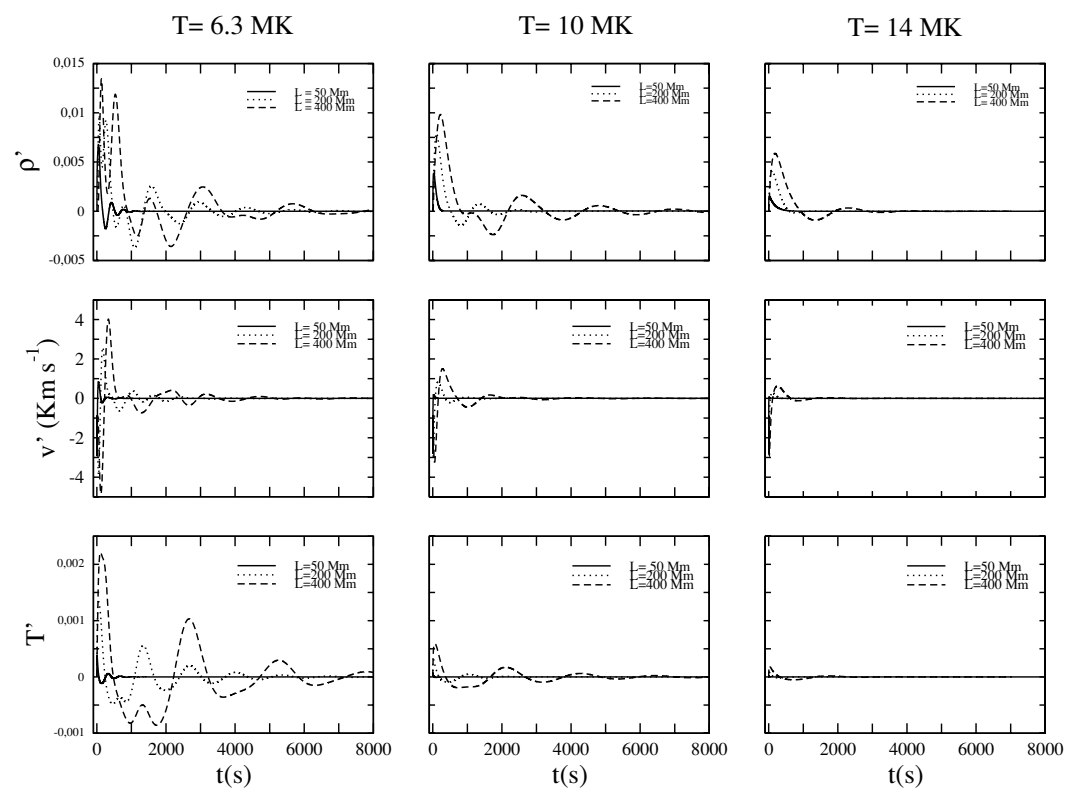

Figure 1. Time evolution of the density, velocity and temperature oscillations at $s=0.35 \mathrm{~L}$ for $s_{0} / L=0.1$ and $\beta_{g} / L=0.01$ in a homogeneous loop for different lengths and temperatures.

where $\chi$ and $\alpha$ are the Hildner's (1974) cooling coefficient and exponent, respectively. The heating rate $H=H(s)$ is set equal to the initial value of $\rho^{2} Q(T)$ so that initially there are no thermal losses. The assumption is also made that the heating rate is kept fixed to its initial value for $t>0$.

\section{Solution Method}

Equations (2.1)-(2.3) are solved in linearized form by expressing the perturbed quantities in terms of a Fourier series expansion, which for a given function $f$, where $f$ may be either $\rho, v, T$, or $p$, has the form:

$$
f(s, t)=\frac{C_{0}(t)}{2}+\sum_{n=1}^{\infty} C_{n}(t) \cos (n \pi s)+\sum_{n=1}^{\infty} \hat{C}_{n}(t) \sin (n \pi s) .
$$

In this way, the temporal and spatial dependence of the perturbed variables is separated and the linearized equations reduce to a set of $6 n$ ordinary differential equations for the time rate of change of the $6 n$ perturbation amplitudes $C_{n}(t)$ and $\hat{C}_{n}(t)$. The time integration is performed numerically using finite-differences for the time derivatives. A predictor-corrector approach is implemented so that the Fourier coefficients (or amplitudes) are calculated with temporal second-order accuracy. We use boundary conditions appropriate for a standing wave problem (De Moortel \& Hood 2003), i.e., all the perturbed variables are set equal to zero at the loop footpoints. Initially, all variables are also set to zero, except the velocity perturbation, which is assumed to take the form

$$
v(s)=v_{0} \exp \left[-\left(\frac{s-s_{0}}{\beta_{g}}\right)^{2}\right],
$$

where $v_{0}=86.683 \mathrm{~km} \mathrm{~s}^{-1}$ is the initial amplitude of the velocity perturbation, $\beta_{g} / L$ is the pulse width (either $0.005,0.01$, or 0.02 ), and $s_{0} / L(=0.1,0.3$, and 0.5$)$ specifies 

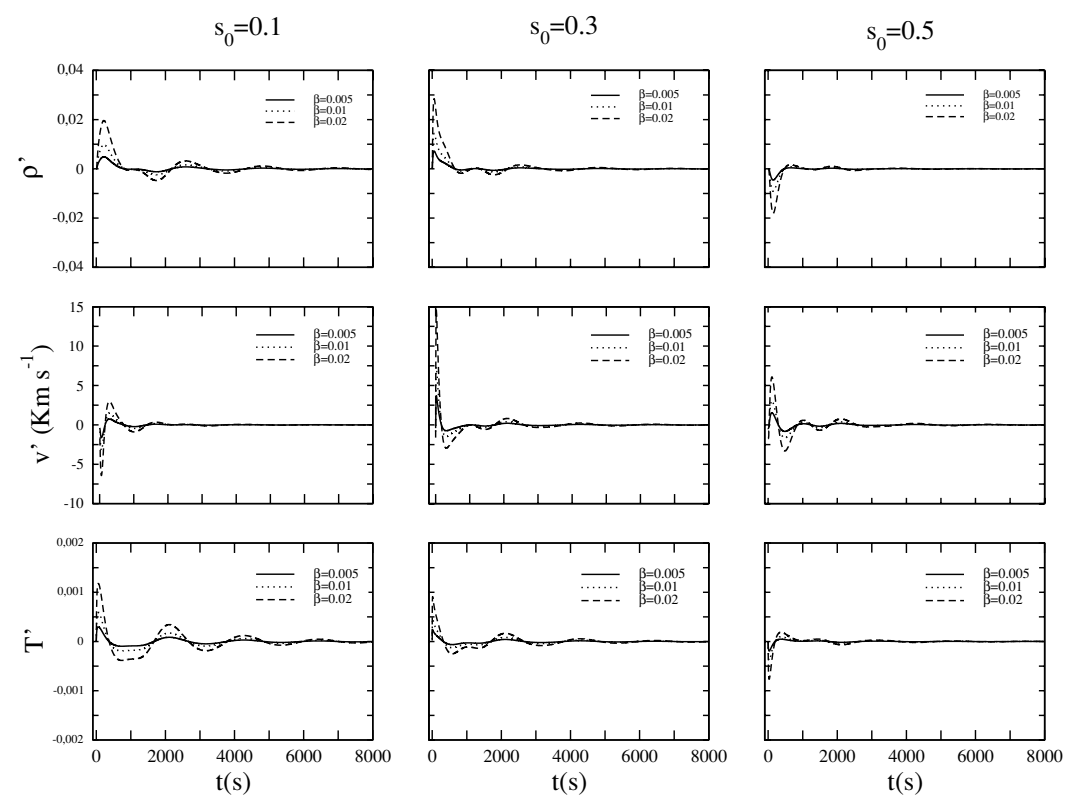

Figure 2. Time evolution of the density, velocity and temperature oscillations at $s=0.35 L$ in a homogeneous loop of $L=400 \mathrm{Mm}$ and temperature $T=10 \mathrm{MK}$ for different values of $\beta_{g} / L$ and $s 0 / L$.

the location of the pulse along the loop, respectively. A value of $s_{0} / L=0.1$ refers to a pulse near the left footpoint, while a value of $s_{0} / L=0.5$ refers to a pulse centered at the loop top. For sufficiently narrow pulses, keeping the velocity perturbation equal to zero at the loop footpoints represent a fairly good approximation. We consider only loops of constant temperature between 6.3 and $14 \mathrm{MK}$ and lengths between 50 and $400 \mathrm{Mm}$.

\section{Results and Conclusions}

Figures 1 and 2 depict the wave evolution at the observation point $\mathrm{s}=0.35 \mathrm{~L}$ for varying initial perturbation and loop parameters. As seen in Figure 1, wave dissipation is significantly more rapid in the shortest $(L=50 \mathrm{Mm})$ and hottest loops $(T=14 \mathrm{MK})$, where the waves effectively decay after about 1 to 2 periods. The wave amplitudes are seen to decrease with increasing loop temperature and decreasing loop length. The irregular wave shapes are a signature of the Gaussian pulse being composed of a large number of harmonic modes.

Figure 2 shows the wave evolutions for different pulse widths and pulse locations at the same observation point as before. Changing the width of the Gaussian pulse does not affect the wave period. However, the wave amplitude increases with the pulse width almost independently of the pulse location along the loop. At a fixed observation point, the wave forms may look differently depending on whether the pulse has been injected near the footpoint or at the loop top. We find that narrower pulses act on the way of producing oscillations that damp out more rapidly because of the lower energy content in the initial perturbation.

We predict wave periods between 0.45 and 10.7 minutes and decay times between 1.52 and 35 minutes. For comparison, De Moortel et al (2002)reported observed periods in the ranges $2.2-10$, for propagating disturbances detected by TRACE. 
We conclude that narrow Gaussian pulses may generate loop oscillations which are quite efficiently dissipated by the effects of thermal conduction and viscosity in both short and long hot coronal loops. The predicted periods and decay times are seen to compare well with those of the observed rapidly decaying modes of hot SUMER loop oscillations. Future work in this line will consider full hydrodynamic simulations to study the nonlinear effects on wave form and wave dissipation. At the same time, it would be interesting to consider the effects of a Gaussian pulse in the initial temperature perturbation distribution on wave generation and damping. This would be equivalent to an energy pulse. On the other hand, a similar analysis to the one presented here shows that for stratified loops the results are not much changed in agreement with previous linear predictions, where the rate of damping is not affected by gravity. Non-equilibrium ionization would be also interesting to be included in the model (Bradshaw and Erdélyi 2007).

\section{Acknowledgements}

C.A.M-B. is grateful to the CDCHT of the Universidad de los Andes for financial support (C-1367-06-05-B). The authors also thank to the IAU for the grant support.

\section{References}

Banerjee, D., Erdélyi, R., Oliver, R., O'Shea, E. 2007, Sol. Phys., 246, 3

Bradshaw, S. J. and Erdélyi, R., 2007 Astron. Astrophys, submitted

Braginskii, S. I. 1965, Rev. Plasma Phys., 1, 205

De Moortel, I., Ireland, J., Walsh, ,R. W. \& Hood, A. W. 2002, Solar Phys., 209, 61

De Moortel, I., \& Hood, A. W. 2003, AESA, 408, 755

Hildner, E. 1974, Solar Phys., 35, 23

Sigalotti, L. Di G., Mendoza-Briceño, C. A., \& Luna-Cardozo, M. 2006, Solar Phys., in press

Taroyan, Y., Erdélyi, R., Wang, T. J. \& Bradshaw, S. J. 2007 ApJ 659, L173 